\title{
Estimativa dos componentes do balanço de energia e da evapotranspiração para áreas de cultivo de soja no sul do Brasil utilizando imagens do sensor TM Landsat 5
}

\author{
Priscylla Ferraz Câmara Monteiro ( $\left.{ }^{* *}\right)$; Denise Cybis Fontana (2); Thiago Veloso dos Santos (3); \\ Débora Regina Roberti $\left(^{4}\right)$
}

(') Fundação Estadual de Pesquisa Agropecuária - Fepagro Nordeste, Centro de Pesquisa da Região Nordeste, Caixa Postal 20, 95200-970 Vacaria (RS), Brasil.

(2) Universidade Federal do Rio Grande do Sul - UFRGS, Faculdade de Agronomia, Departamento de Plantas Forrageiras e Agrometeorologia, Av. Bento Gonçalves, 7712, 91540-000 Porto Alegre (RS), Brasil.

(3) University of Minnesota, Department of Soil, Water, and Climate, 439 Borlaug Hall, 1991 Upper Buford Circle, 551086112 Saint Paul (USA).

(4) Universidade Federal de Santa Maria, Centro de Ciências Naturais e Exatas, Departamento de Física, Av. Roraima, 1000, $97105-$ 900 Santa Maria (RS), Brasil.

(*) Autora correspondente: priscylla-monteiro@fepagro.rs.gov.br

Recebido: 20/jun./2013; Aceito: 5/fev./2014

\section{Resumo}

O presente estudo teve como objetivo avaliar a eficiência do algoritmo SEBAL na estimativa dos componentes de balanço de energia e da evapotranspiração para áreas de cultivo de soja irrigada no município de Cruz Alta (RS), assim como analisar a variação temporal e espacial da evapotranspiração a partir de imagens do sensor TM Landsat 5. O algoritmo Surface Energy Balance Algorithm for Land (SEBAL) foi aplicado a seis imagens distribuídas ao longo do ciclo fenológico da cultura da soja, no município de Cruz Alta, RS, nas safras 2009 e 2010. As estimativas obtidas foram comparadas com os dados de superfície, obtidos por meio da estação micrometeorológica localizada na área de estudo. As estimativas mais precisas foram as do saldo de radiação, enquanto que a estimativa que apresentou maior erro foi a do fluxo de calor no solo. O SEBAL mostrou potencial para mapear a variabilidade espacial e temporal da evapotranspiração, evidenciando sensibilidade aos diferentes tipos de ocupação do solo e aos diferentes estádios de desenvolvimento da cultura.

Palavras-chave: Glycine max L., sensoriamento remote, SEBAL, recursos hídricos.

\section{Estimation of energy balance components and evapotranspiration in soybean crop in southern Brazil using TM - Landsat 5 images}

Abstract

The present study aimed to evaluate the efficiency of SEBAL algorithm in estimating the energy balance components and evapotranspiration for irrigated soybean areas, near the municipality of Cruz Alta, RS, Brazil, and to analyze the temporal and spatial variation of evapotranspiration from Landsat TM5 image. The Surface Energy Balance Algorithm for Land (SEBAL) was applied to six images distributed throughout the soybean growth season in 2009 and 2010 harvests. The estimates were compared to micrometeorological data retrieved from a station located in the studied area. The most accurate modeled parameter estimatives was net radiation while the one which presented the highest error was soil heat flux. The SEBAL showed potential to map the spatial and temporal evapotranspiration variability, showing sensitivity to different types of land cover and to different crop development stages.

Key words: Glycine max L., remote sensing, SEBAL, water resources. 


\section{INTRODUÇÃO}

A agricultura é a base da economia do estado do Rio Grande do Sul e as culturas da soja e do milho são as de maior destaque. Na safra 2010/2011, a área colhida para essas duas culturas foi de 4,085 e 1,099 milhão de hectares, com uma produçáo de 11,621 e 5,776 milhôes de toneladas e produtividade média de 2.845 e $5.255 \mathrm{~kg} \mathrm{ha}^{-1}$, respectivamente (CONAB, 2012). A soma da participação dessas duas culturas representa mais de $70 \%$ da área cultivada e elas respondem por mais de $60 \%$ da produção total de grãos do estado (CONAB, 2012).

Tendo em vista que praticamente a totalidade de grãos (exceção para a cultura do arroz irrigado) produzidos no estado é oriunda de lavouras de sequeiro, a produtividade e a produção apresentam alta variabilidade interanual, determinada principalmente pela variabilidade da precipitação pluvial (Berlato e Fontana, 1999). Para quantificar a demanda hídrica de uma cultura é necessário conhecer o ciclo hidrológico e, principalmente, a componente evapotranspiração. A quantificação do processo de evapotranspiraçáo normalmente é feita utilizando-se equipamentos como lisímetros ou, ainda, através de equaçóes de estimativa, como a de Penman-Monteith (Allen et al., 1998), e, mais recentemente, pelo método eddy covariance, que utiliza medidas atmosféricas em alta frequência. Porém, esses métodos estimam valores de evapotranspiraçáo para um local específico e não podem ser extrapolados para uma ampla escala espacial (Neves et al., 2007).

Levando em conta essas limitaçóes e o alto custo de instalação e manutenção de complexos equipamentos de medição, o sensoriamento remoto passa a ser uma importante ferramenta para a rápida obtenção de dados hidrológicos, permitindo estimativas em escala regional com baixo custo operacional.

Alguns algoritmos baseados em sensoriamento remoto foram desenvolvidos nos últimos anos para simplificar o processo de obtenção de variáveis hidrológicas para extensas regióes geográficas (Bastiaanssen et al., 1998). O SEBAL (Surface Energy Balance Algorithm for Land - Bastiaanssen et al., 2005), empregado no presente trabalho, leva em consideração a variabilidade espacial de elementos micrometeorológicos por meio de funçóes semiempíricas e pode ser aplicado em diferentes agrossistemas. Esse algoritmo estima a evapotranspiração em larga escala geográfica, utilizando imagens de satélite e alguns dados complementares de superfície, como temperatura do ar e velocidade do vento.

$\mathrm{Na}$ literatura científica demostra-se a potencialidade e capacidade de diversos tipos de sensores remotos, com diferentes métodos de calibração, de estimar os componentes do balanço de energia e da evapotranspiração utilizando-se informaçôes orbitais nas bandas do infravermelho e do termal (Santos et al., 2010). Silva e Bezerra (2006), em estudo de quantificaçáo dos componentes do balanço de energia utilizando imagens TM Landsat 5 e o algoritmo SEBAL, observaram variaçóes entre $1 \%$ e $5 \%$ quando comparadas as medidas assim obtidas às de campo; e, French et al. (2005), que utilizaram o sensor ASTER e avaliaram os fluxos de energia em superfície sobre diferentes culturas agrícolas, apresentaram resultados com variaçôes superiores a $20 \%$.

Santos et al. (2010) mapearam os fluxos de calor em superfície, no Rio Grande do Sul, por meio de dados orbitais, utilizando os sensores AVHRR/NOAA e ASTER. Devido à carência de dados para o estado, os autores ressaltaram a relevância de testar a potencialidade de sensores dotados com diferentes resoluçôes espacial e temporal. Com isso, como os dados do satélite Landsat, integrados ao modelo SEBAL, já apresentaram resultados satisfatórios em outras regióes, essa pode ser uma alternativa para ampliação das informaçôes desejadas no estado.

A presente pesquisa teve por objetivo avaliar a eficiência do algoritmo SEBAL na estimativa de componentes do balanço de energia tais como: saldo de radiação, fluxos de calor no solo sensível e latente e a evapotranspiração para áreas de cultivo de soja irrigada na região de Cruz Alta, RS, e na análise da variação espaço-temporal da evapotranspiração a partir de imagens do sensor TM Landsat 5.

\section{MATERIAL E MÉTODOS}

A área de estudo está localizada no município de Cruz Alta, situado na região noroeste do estado do Rio Grande do Sul, entre as coordenadas $28^{\circ} 25^{\prime} 22^{\prime \prime}$ a $28^{\circ} 57^{\prime} 51^{\prime \prime} \mathrm{S}$ e $53^{\circ} 53^{\prime} 46^{\prime \prime}$ a $53^{\circ} 14^{\prime} 04^{\prime \prime}$ W e a cultura predominante da região, no período de primavera-verão, é a soja.

A semeadura da soja na área experimental, nas safras de 2008 e 2009, no município de Cruz Alta, RS, foi realizada no dia 28 de novembro e 10 de dezembro, respectivamente, de acordo com a época de semeadura da região e respeitando o zoneamento agrícola do estado, que para essa região determina o período de $1 .^{\circ}$ de novembro a 31 de dezembro. O ciclo da soja disponível no mercado brasileiro varia entre 100 e 160 dias, dependendo da variedade utilizada.

A precipitação pluvial média anual da região é de cerca de $1.800 \mathrm{~mm}$ e as temperaturas médias anuais situam-se entre $13,3{ }^{\circ} \mathrm{C}$, no mês mais frio, e $24,6{ }^{\circ} \mathrm{C}$, no mês mais quente (CEMETRS, 2011). O clima da região enquadrase na categoria Cfa, segundo a classificação de Köppen, ou seja, subtropical úmido com veróes quentes. Os solos predominantes na região são os Latossolos Vermelhos Distróficos.

Os dados meteorológicos de superfície utilizados, temperatura do ar $\left({ }^{\circ} \mathrm{C}\right)$ e velocidade do vento $\left(\mathrm{m} \mathrm{s}^{-1}\right)$, provenientes da estação meteorológica do INMET, foram coletados nos mesmos horários da passagem do satélite, para realização da validação do SEBAL. Essa estação está 
instalada nas dependências da empresa CCGL-TEC/ FUNDACEP (2860’36” S; 5367’36” W; 436 m), em um sítio experimental de cultivo agrícola localizado no município de Cruz Alta, RS.

As cenas utilizadas foram geradas pelo sensor TM (Thematic Mapper) a bordo do satélite Landsat 5, correspondentes a órbita-ponto 223-80 e distribuídas pela Divisão de Geração de Imagens (DGI) do Instituto Nacional de Pesquisas Espaciais (INPE). As datas de tomada das imagens foram, em 2009, 5/1, 21/1, 26/3 e 11/4 e, em 2010, 24/1 e 9/2, as quais referem-se aos Dias Julianos (DJ) $5,21,85,101,24$ e 40, respectivamente. Essas cenas foram selecionadas por apresentarem baixa cobertura de nuvens, com o objetivo de apoiar uma análise temporal consistente da evapotranspiração sobre a região do estudo. Cada cena do sensor TM Landsat 5 possui dimensão de $185 \mathrm{~km} \times$ $185 \mathrm{~km}$ e resolução espacial de $30 \mathrm{~m}$ para as bandas visível e infravermelho e de $120 \mathrm{~m}$ para a banda do termal.

As etapas para execução do algoritmo SEBAL estão descritos com detalhes em Allen et al. (2005). Inicialmente, os números digitais da imagem foram convertidos para radiância utilizando-se os parâmetros recomendados por Chander et al. (2007). Correçôes atmosféricas baseadas no modelo de transferência radiativa 6S (Tanré et al., 1990) foram aplicadas nas imagens de radiância com o objetivo de remover os efeitos de absorção, espalhamento e reflexão que a atmosfera exerce sobre a radiação, obtendo, assim, as imagens de reflectância de superfície.

O saldo de radiação $\left(R_{\mathrm{n}}\right)$, que indica a radiação disponível em superfície, é derivado a partir do albedo e da emissividade da superfície, assim como de parâmetros orbitais que variam em função da distância Terra-Sol.

O fluxo de calor no solo $(G)$ é estimado por meio da aplicação de uma equação empírica proposta por Bastiaanssen (2000), que leva em conta propriedades da superfície tais como temperatura, albedo e tipo de cobertura (expresso pelo NDVI).

A determinação do fluxo de calor sensível $(H)$ é a etapa mais importante do SEBAL, consistindo num processo iterativo que envolve a resistência aerodinâmica da superfície, estimada a partir de um registro de velocidade do vento e da localizaçáo na imagem de pontos chamados pixels-âncora. Esses pixels refletem duas condiçóes extremas de fluxo de calor: a) o "pixel frio" é selecionado sobre áreas bem irrigadas, em condiçôes ideais de evapotranspiração ou em grandes lâminas de água, onde o fluxo de calor sensível é considerado nulo; b) o "pixel quente" é selecionado sobre áreas sem vegetaçáo fotossinteticamente ativa e sem umidade, como solo exposto em condições críticas de evapotranspiração, assumindo que o fluxo de calor latente é considerado nulo nessas áreas (Bastiaanssen et al., 1998). Maiores detalhes sobre os critérios de escolha dos pixels-âncora podem ser consultados em Santos et al. (2010).
O algoritmo SEBAL utiliza a equação do balanço de energia à superfície (Equaçáo 1) para estimar o fluxo de calor latente. Dessa forma, $L E$ é descrito como um resíduo para o fechamento do balanço de energia através de $R n, G$ e $H$.

$\mathrm{LE}=\mathrm{Rn}-\mathrm{G}-\mathrm{H}$

em que: $L E$ é expresso em unidades de $\mathrm{W} \mathrm{m}^{-2}$.

$\mathrm{O} L E$ é convertido em evapotranspiração horária, de acordo com a expressão (Equação 2):

$\mathrm{ET}_{\mathrm{h}}=1.000(L E / \mathrm{\rho} \lambda) \Delta t$

em que: $E T_{\mathrm{h}}$ é a evapotranspiração horária estimada para a hora da passagem do satélite; $\lambda$ é o calor latente de vaporizaçáo da água $\left(\lambda=2,45 \times 10^{6} \mathrm{~J} \mathrm{~kg}^{-1}\right) ; \rho$ é a densidade da água $\left(\rho=1.000 \mathrm{~kg} \mathrm{~m}^{-3}\right) ; \Delta t$ é o intervalo de tempo usado, no caso 3.600 s; e o valor 1.000 é a conversão para que a evapotranspiração seja expressa em $\mathrm{mm} \mathrm{dia}^{-1}$.

A aproximadamente $400 \mathrm{~m}$ da estação meteorológica, uma torre de fluxo, pertencente ao projeto SULFLUX (www.ufsm. br/sulflux) coleta dados de alta frequência de velocidade do vento e de temperatura, através de um anemômetro sônico 3D, modelo CSAT3 (Campbell Scientific Inc., Logan, Utah, USA), e de vapor d'água, através do analisador de gás modelo LI-7500 (LI-COR Corporate Offices, Lincoln, Nebrasca, USA), a $3 \mathrm{~m}$ de altura. Os fluxos de calor sensível e latente são calculados através desses dados utilizando-se o método eddy covariance (Baldocchi et al., 1988). Dados de saldo de radiação (sensor NR LITE, Kipp \& Zonen), a 2 m, e fluxo de calor no solo (sensor HFP01SC-L - Hukseflux), a 0,05m, também foram coletados na torre de fluxo. Informaçóes adicionais sobre a área experimental e as medidas de fluxo podem ser obtidas em Webler et al. (2012).

Para a visualização e realização de operaçôes matemáticas intra e interbandas, recortes, classificação e outras operações, utilizou-se o software ERDAS Imagine 9.1. Antes de iniciar o processo de tratamento digital de imagens propriamente dito, foi realizado um pré-processamento, que consistiu no empilhamento das bandas, recorte, reamostragem da banda $6(10,4-12,5 \mu \mathrm{m})$ e correçóes geométricas, processos importantes para a melhoria da qualidade dos dados (Versiani e Rodrigues, 2007).

Para viabilizar as análises deste trabalho, as seguintes classes de alvos foram selecionadas: a) vegetação nativa, b) culturas anuais, c) área urbana e d) água. Para a classe culturas anuais, a cultura selecionada para análise nas duas safras foi a soja em áreas irrigadas, sendo esse termo utilizado apenas para definir a classe e indicar que essas áreas podem ser ocupadas por outras culturas em diferentes safras. Para cada classe foram construídos polígonos chamados regiōes de interesse (Rois), os quais foram restaurados para as diferentes datas. As áreas selecionadas foram representadas pela média de pixels contida nas Rois. O dimensionamento do número de pixels e da área (ha) que integraram a Roi para cada alvo utilizado para as análises podem ser observados 
na tabela 1. Culturas anuais e vegetação nativa tiveram uma área amostrada superior às demais.

\section{RESULTADOS E DISCUSSÃO}

As componentes do balanço de energia (saldo de radiação, $H, L E$, e $G$ ) foram estimadas com a simulação do modelo SEBAL utilizando-se imagens de satélite para a descrição da superfície e dados observados de temperatura e velocidade do vento. Os resultados e comparaçóes com medidas observacionais são descritos a seguir.
A figura 1a-d apresenta a série temporal do valor médio dos pixels que compóem cada um dos alvos amostrados para os componentes do balanço de energia, obtidos no momento da passagem do satélite, nas duas safras. O saldo de radiação representa a energia disponível para os processos físicos e biológicos que ocorrem na superfície terrestre. Todos os alvos analisados, em 2009 e em 2010, apresentaram padrão similar de evoluçáo temporal, com valores altos no período de maior disponibilidade de radiação solar (janeiro e fevereiro), quando ocorreram as maiores diferenças em $R n$, conforme esperado, e que diminuiram à medida que a disponibilidade de radiação solar decresceu (março e abril). A diferença na magnitude de $R n$ entre os alvos está associada ao albedo da

Tabela 1. Alvos selecionados na área de estudo, no município de Cruz Alta, RS, contendo o número de pixel e o tamanho da área (ha) das regióes de interesse para as safras de 2009 e 2010

\begin{tabular}{|ccccc|}
\hline Alvos & & $\mathbf{2 0 0 9}$ & \multicolumn{2}{c}{$\mathbf{2 0 1 0}$} \\
\hline Vegetação nativa & No de pixels & Área (ha) & No de pixels & Área (ha) \\
\hline Culturas anuais & 3.325 & 299,25 & 2.437 & 219,33 \\
\hline Áreas urbanas & 5.424 & 488,16 & 3.714 & 334,26 \\
\hline Água & 488 & 43,92 & 488 & 43,92 \\
\hline
\end{tabular}
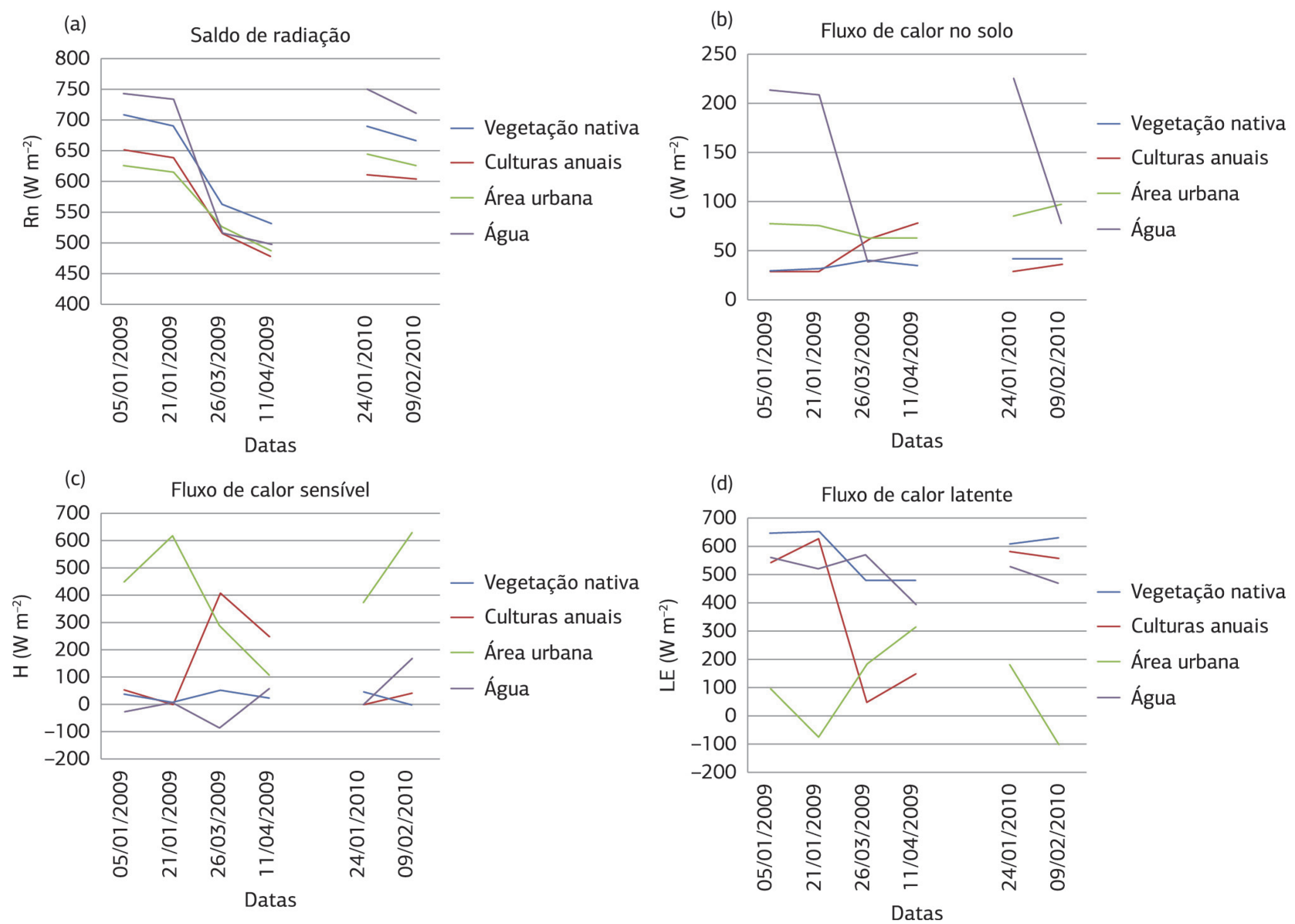

Figura 1. Variação das médias dos pixels dos componentes do balanço de energia obtidos em áreas de vegetação nativa, áreas de cultura agrícola, área urbana e corpos d'água nos dias 5 e 21 de janeiro, 26 de março e 11 de abril de 2009 e nos dias 24 de janeiro e 9 de fevereiro de 2010. 
superfície, que é o principal termo de perda de radiação da superfície, característico de cada alvo.

Os maiores valores de $R n$ foram observados nos corpos d'água. A água, entre os corpos naturais, é aquele que apresenta a maior absorção da radiação incidente e, portanto, o menor albedo. Valores típicos de albedo em corpos d'água variaram de $3 \%$ a $12 \%$, similare aos encontrados por Santos et al. (2010) e Silva et al. (2005). Observou-se, neste estudo, que os valores de $R n$ variaram de $750 \mathrm{~W} \mathrm{~m}^{-2}$ a $500 \mathrm{~W} \mathrm{~m}^{-2}$, de janeiro a abril de 2009 , e de $750 \mathrm{~W} \mathrm{~m}^{-2}$ a $711 \mathrm{~W} \mathrm{~m}^{-2}$, de janeiro a fevereiro de 2010. Todos os alvos analisados apresentaram, apesar das diferenças, tendência de variação temporal semelhante. Entretanto, quando se analisaram os destinos do $R n$, ou seja, a partição da energia disponível entre $G, H$ e $L E$, diferenças importantes foram verificadas entre os alvos.

A área de vegetação nativa apresentou valores de $R n$ maiores do que $700 \mathrm{~W} \mathrm{~m}^{-2}$ nas duas primeiras datas de 2009 e de 2010 (Figura 1a). Os valores encontrados são coerentes e na mesma ordem de magnitude dos encontrados por Bezerra et al. (2008) e Santos et al. (2010) para áreas com cobertura vegetativa de densidade intermediária (entre $678 \mathrm{~W} \mathrm{~m}^{-2}$ e $703 \mathrm{~W} \mathrm{~m}^{-2}$ ). Em março e abril de 2009 houve nesse alvo reduçáo em $R n$, que atingiu valores próximos de $550 \mathrm{~W} \mathrm{~m}^{-2}$. Essa redução está associada à diminuição de biomassa verde, comum nesse período do ano. Portanto, também mostram coerência com os valores encontrados por Bezerra et al. (2008), em torno de $600 \mathrm{~W} \mathrm{~m}^{-2}$ quando a vegetação nativa apresentava menor densidade, muito embora para regiáo diferente da aqui estudada.

Observa-se que o $R n$ da área ocupada pela classe culturas anuais selecionadas em áreas de pivô central e representada pela cultura da soja apresentou valores inferiores ao campo nativo em todos os dias da análise. Esse resultado pode náo parecer coerente com o esperado, dado que o albedo apresenta uma relação inversa com a altura da vegetação - quanto maior a altura, menor é o valor do albedo (consequência das reflexôes múltiplas no interior do dossel, causando maior absorção da radiaçáo incidente). Assim, a cultura da soja irrigada, com maior porte, deveria ter menor albedo e, portanto, maior $R n$. Uma possível explicação para o resultado obtido neste estudo em 2009 está associada ao período de aquisição das imagens. Em janeiro, a soja ainda está no período de estabelecimento e, portanto, a biomassa verde ainda é baixa, possivelmente menor do que a da vegetaçáo nativa. $\mathrm{O}$ mesmo ocorre em final de março e em abril.

$\mathrm{O}$ alvo área urbana apresentou valores abaixo de $650 \mathrm{~W}$ $\mathrm{m}^{-2} \mathrm{em}$ todos os dias estudados. Tal padrão é esperado devido à baixa quantidade de vegetação e à alta impermeabilização do solo, apresentando maior albedo, ou seja, reflete mais energia em onda curta, resultando numa menor absorção da radiação incidente.

Como fração do saldo de radiaçáo, o fluxo de calor no solo $(G)$ representa a parte da energia que é transferida para os níveis inferiores do solo e, geralmente, resulta em acréscimo de energia para o meio à noite, ou em diminuição no período diurno (Galvani et al., 2001). O fluxo de calor no solo é função da temperatura em diferentes profundidades e da condutividade térmica do solo. Ao analisar o padrão dos valores de $G$ (Figura $1 \mathrm{~b}$ ) obtidos neste estudo, observase que a quantidade de energia destinada ao aquecimento do solo foi muito inferior àquela destinada para $H$ ou $L E$. Os valores de menor magnitude de $G$ ocorreram nas áreas úmidas (vegetação nativa e culturas anuais). Os corpos d'água apresentaram os maiores valores para essa variável no mês de janeiro, até mesmo acima dos valores encontrados nas áreas urbanas e nas áreas desprovidas de vegetação, fato esse decorrente do calor específico da água. Rodrigues et al. (2009), empregando imagens do Landsat 7 , obtiveram valores superiores a $190 \mathrm{~W} \mathrm{~m}^{-2}$ para corpos d'água. A área urbana se comportou como uma ilha de calor, apresentando, coerentemente, um fluxo de calor elevado quando comparada com outros alvos (Weng et al., 2004). Neste estudo, os valores variaram de $77 \mathrm{~W} \mathrm{~m}^{-2}$ a $62 \mathrm{~W} \mathrm{~m}^{-2}$, de janeiro a abril de 2009 , e de $85 \mathrm{~W} \mathrm{~m}^{-2}$ a $96 \mathrm{~W} \mathrm{~m}^{-2}$, nos meses de janeiro e fevereiro de 2010. A quantidade de energia utilizada para o aquecimento do solo no alvo área urbana foi de aproximadamente $12 \%$ de $R n$ para as diferentes datas analisadas. Em abril de 2009, os pixels que representam a cultura da soja passaram a ser ocupados por solo exposto devido à colheita já ter sido realizada na região. As regiōes de solo exposto apresentaram os índices mais elevados de fluxo de calor no solo quando comparadas aos alvos área urbana e vegetaçáo nativa, com valores em torno de $76 \mathrm{~W}$ $\mathrm{m}^{-2}$, aproximadamente $16 \%$ de $R n$. A vegetação tende a atenuar a transferência de energia para o solo (Jensen, 2007). Santos et al. (2010) encontraram valores superiores a $14 \%$ em regióes desprovidas de cobertura ou com vegetaçáo nativa rala.

O fluxo de calor sensível $(H)$ representa a parte da energia disponível na superfície que é transferida para a atmosfera, para a variação da sua temperatura. Na figura 1c observase que os menores valores de $H$ correspondem aos pixels contendo massas de água. Tanto em 2009 como em 2010, esse alvo apresentou valores próximos a zero ou negativos. As regióes urbanizadas, no caso a cidade de Cruz Alta, RS, foram marcadas por valores de calor sensível entre $114 \mathrm{~W}$ $\mathrm{m}^{-2}$ e $629 \mathrm{~W} \mathrm{~m}^{-2}$, os de maior magnitude entre os alvos analisados. Santos et al. (2010) encontraram a faixa de $200 \mathrm{~W} \mathrm{~m}^{-2}$ a $400 \mathrm{~W} \mathrm{~m}^{-2}$ para regiāo urbana de Paraíso do Sul, RS, em 4 de março de 2004. Os valores mais elevados de calor sensível encontram-se nas regiōes de menor cobertura vegetativa (com baixos valores de NDVI), ou seja, áreas urbanas e solo exposto. Tal comportamento justifica os menores valores encontrados para a vegetação nativa, entre $3 \mathrm{~W} \mathrm{~m}^{-2}$ e $49,7 \mathrm{~W} \mathrm{~m}^{-2}$, e culturas anuais, entre $1,4 \mathrm{~W} \mathrm{~m}^{-2}$ e 249,7 W m${ }^{-2}$, com exceção dos meses de março e abril, em que os valores chegam a 406,4 $\mathrm{W} \mathrm{m}^{-2}$ e $249,7 \mathrm{~W} \mathrm{~m}^{-2}$, 
respectivamente. Recorda-se que nessa época grande parte da soja já havia sido colhida e que esse alvo passa a dar espaço ao solo exposto.

O fluxo de calor latente $(L E)$ representa a parte da energia utilizada na mudança de fase da água. Em janeiro, as áreas compostas por vegetação nativa foram as que apresentaram os maiores valores de $L E$, acima de $608 \mathrm{~W} \mathrm{~m}^{-2}$ (Figura $1 \mathrm{~d}$ ). Os valores são diretamente proporcionais à densidade da vegetação, decrescendo bruscamente nas culturas anuais, em funçáo da senescência das plantas, e mais lentamente na vegetação nativa, em função da diminuição da demanda evaporativa da atmosfera, dada a declinação solar. Corroborando essa afirmaçáo, as regiôes de solo exposto e área urbana apresentam os menores valores de $L E$.

A $L E$ de lavouras irrigadas em início de desenvolvimento assemelha-se ao observado sobre as massas de água. A soja instalada em área irrigada por pivô central apresentou valores de $L E$ da ordem de $618 \mathrm{~W} \mathrm{~m}^{-2}$ e $580 \mathrm{~W} \mathrm{~m}^{-2}$ para os meses de fevereiro de 2009 e janeiro de 2010, respectivamente, quando a cultura estava em pleno desenvolvimento vegetativo, o que representou aproximadamente $85 \%$ e $95 \%$ de $R n$, respectivamente. Resultados bastante similares a esses foram encontrados por Bastiaanssen (2000) para a cultura do algodáo em áreas semiáridas da Turquia - em torno de $85 \%$ de $R n$.

A comparação das componentes do balanço de energia estimadas pelo modelo SEBAL e as medidas observacionais são apresentadas na tabela 2. Para o saldo de radiação, as medidas observacionais e as estimativas foram muito similares. Os valores estimados foram sempre superiores aos medidos, com um erro médio de $8 \%$ e um coeficiente de correlação de 0,992 , o que demonstra forte associação entre os dois métodos de obtenção de $R n$. Já para a partição do balanço de energia foram observadas diferenças importantes em alguns dos dias avaliados. O fluxo de calor para o solo foi o componente em que se observou a menor associação entre medida e estimativa $(r=0,386)$, havendo dias tanto de superestimativa como de subestimativa.

Para $H$ e $L E$, os coeficientes de correlação foram, respectivamente, 0,730 e 0,756 , ambos significativos a $90 \%$ de significância. A energia destinada ao fluxo de calor latente foi o componente com menores diferenças nas estimativas. Dessa forma, o SEBAL pode ser utilizado para gerar mapas de evapotranspiração com boa precisão, pois ela é obtida diretamente do fluxo de calor latente (conforme descrito na seçấo SEBAL). Ressalta-se ainda que a metodologia utilizada estima parâmetros biofísicos em escala regional e que, portanto, a validaçấo com os dados de torre, no nosso caso, serve para verificar a coerência dos resultados e não para validaçáo extensiva.

A evapotranspiração das culturas agrícolas depende da demanda evaporativa da atmosfera, das condiçôes hídricas do solo e do estádio de desenvolvimento fenológico da cultura. No início do desenvolvimento da cultura, a evapotranspiração, devido à baixa porcentagem de cobertura vegetal, é composta principalmente de evaporação das superfícies d'água. À medida que a cultura se desenvolve, a taxa de evaporaçáo

Tabela 2. Estatísticas dos componentes do balanço de energia (valores médios medidos e estimados (W $\mathrm{m}^{-2}$ ), diferença da estimativa (\%), fração do $R n$ (saldo de radiação) utilizado em $G$ (fluxo de calor sensível no solo), $H$ (fluxo de calor sensível no ar) e $\lambda E T$ (fluxo de calor latente), coeficiente de correlação e significância) obtidos em lavoura experimental de soja no município de Cruz Alta, RS, nas safras 2009 e 2010

\begin{tabular}{|c|c|c|c|c|c|c|c|c|}
\hline \multicolumn{9}{|c|}{ Datas } \\
\hline & & 2009 & & & 2010 & & & \\
\hline & $5 / 1 / 2009$ & $21 / 1 / 2009$ & $26 / 3 / 2009$ & $11 / 4 / 2009$ & $24 / 1 / 2010$ & $9 / 2 / 2010$ & Correlação & a \\
\hline \multicolumn{9}{|c|}{ Rn } \\
\hline Medido & 626,15 & 608,38 & 460,86 & 426,55 & 603,07 & 572,19 & & \\
\hline Estimado & 643,56 & 627,95 & 517,44 & 509,68 & 616,00 & 611,35 & 0,992 & $0,01 \%$ \\
\hline Dif. (\%) & 3 & 3 & 12 & 19 & 2 & 7 & & \\
\hline \multicolumn{9}{|c|}{ G } \\
\hline Medido & 115,16 & 37,73 & 22,76 & 41,66 & 84,20 & 21,82 & & \\
\hline Estimado & 65,37 & 39,14 & 35,66 & 62,52 & 53,16 & 66,39 & 0,386 & NS \\
\hline Dif. (\%) & 43 & 4 & 57 & 50 & 37 & 204 & & \\
\hline $\mathrm{G} / \mathrm{Rn}$ & 10 & 6 & 7 & 12 & 9 & 11 & & \\
\hline \multicolumn{9}{|c|}{$\mathbf{H}$} \\
\hline Medido & 176,55 & 112,67 & 136,90 & 27,19 & 123,29 & 78,33 & & \\
\hline Estimado & 352,13 & 156,23 & 123,91 & 110,21 & 146,11 & 64,31 & 0,730 & 0,10 \\
\hline Dif. (\%) & 99 & 38 & 10 & 305 & 18 & 18 & & \\
\hline $\mathrm{H} / \mathrm{Rn}$ & 55 & 25 & 24 & 22 & 24 & 10 & & \\
\hline \multicolumn{9}{|c|}{ ET } \\
\hline Medido & 264,01 & 318,83 & 239,18 & 248,02 & 392,57 & 469,76 & & \\
\hline Estimado & 226,06 & 432,57 & 357,87 & 336,95 & 416,72 & 480,65 & 0,7556 & 0,10 \\
\hline Dif (\%) & 14 & 35 & 50 & 36 & 6 & 2 & & \\
\hline $\mathrm{ET} / \mathrm{Rn}$ & 35 & 69 & 69 & 66 & 67 & 79 & & \\
\hline
\end{tabular}



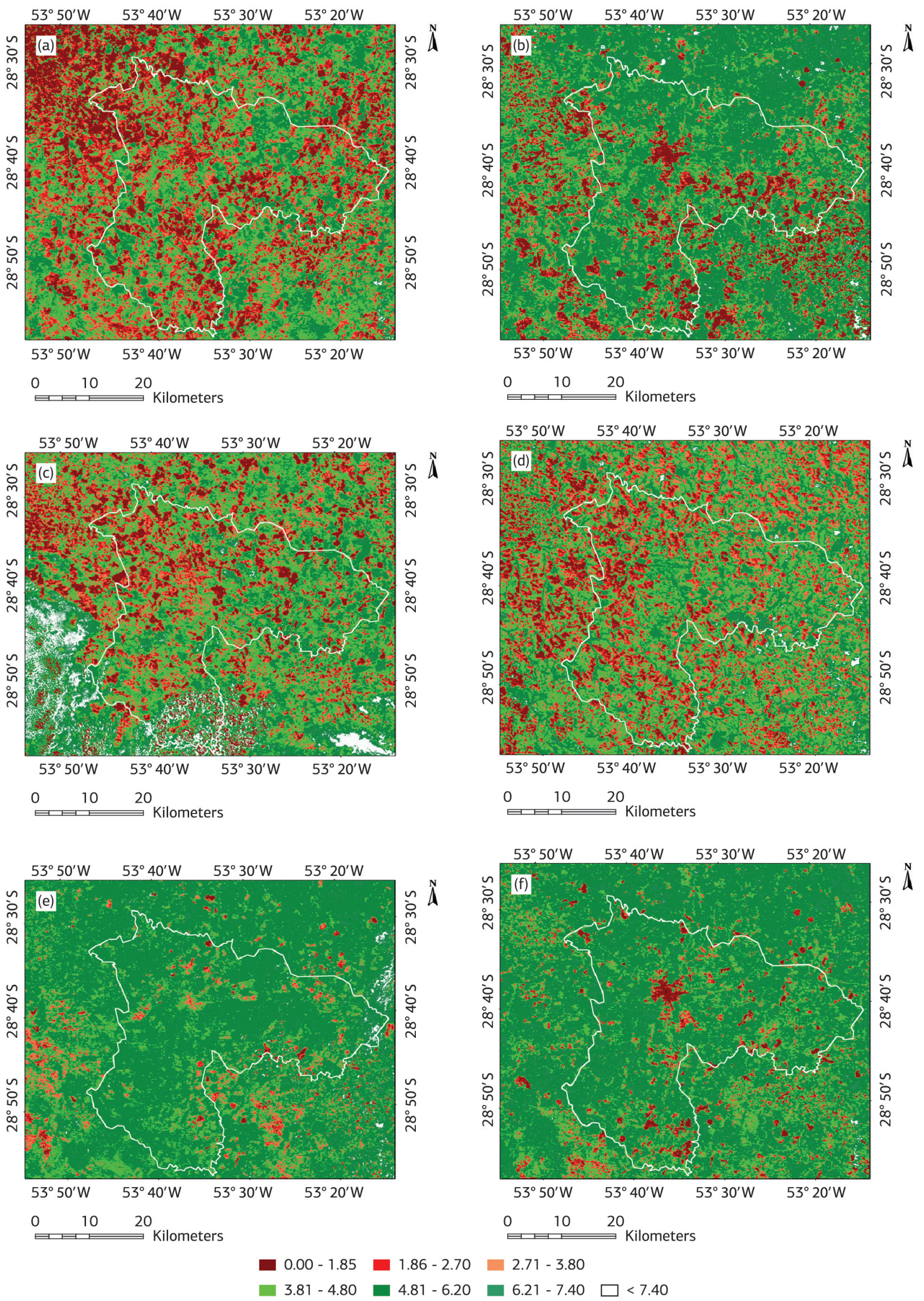

Figura 2. Distribuição espacial da evapotranspiração diária (mm dia-1), nos dias: (a) 5/1/2009; (b) 21/1/2009; (c) 26/3/2009; (d) 11/4/2009; (e) $24 / 1 / 2010$; e (f) $9 / 2 / 2010$. 
decresce e a transpiração do dossel aumenta, fazendo com que a evapotranspiração da cultura aumente (Allen et al., 1998).

Berlato et al. (1986), avaliando o consumo de água pela cultura da soja, no Rio Grande do Sul, obtiveram um consumo médio diário de $5,8 \mathrm{~mm}$. Esse consumo, expresso pela evapotranspiração diária variou de $2,2 \mathrm{~mm}$, no subperíodo plantio-emergência, até o valor máximo de $7,4 \mathrm{~mm}$, no estádio do início do florescimento até o início do enchimento de grãos. O mapeamento das taxas de evapotranspiração no município de Cruz Alta, segundo a metodologia SEBAL (Figura 2 a-f), apresenta concordância com esses valores. As lavouras de soja, predominantemente marcadas com a cor verde-claro e verde intermediário, apresentaram evapotranspiração diária entre $5 \mathrm{~mm} \mathrm{dia}^{-1}$ e $6,5 \mathrm{~mm} \mathrm{dia}^{-1}$ nos meses de janeiro e fevereiro (2009 e 2010), correspondendo ao período de pleno desenvolvimento vegetativo. Em contrapartida, os menores valores de evapotranspiração foram encontrados em 26 de março e 11 de abril de 2009, com média de $2,3 \mathrm{~mm} \mathrm{dia}^{-1} \mathrm{e}$ $3,2 \mathrm{~mm} \mathrm{dia}^{-1}$, respectivamente. Essas datas corresponderam ao estádio de maturação da cultura e semeadura de outras culturas e, portanto, apresentam baixa quantidade de biomassa verde e maior quantidade de solo exposto.

As áreas de solo exposto e área urbana, marcadas com a cor vermelha e coral nas imagens, apresentaram os menores valores de ET, com valores inferiores a 3,4 $\mathrm{mm} \mathrm{dia}^{-1}$. As áreas com vegetação nativa, destacadas em cor verde mais escura, apresentaram valores de ET entre $6,4 \mathrm{~mm} \mathrm{dia}^{-1} \mathrm{e}$ $7,4 \mathrm{~mm} \mathrm{dia}^{-1}$.

As massas de água, apesar de não identificáveis na Figura 2 em função da escala e representação e do tamanho dos corpos d'água validados, mostraram valores diários de ET acima de $7,2 \mathrm{~mm} \mathrm{dia}^{-1}$ para todas as datas, com exceção dos dias $9 / 2 / 2010$ e 11/4/2009, que apresentaram valores entre 5,7 $\mathrm{mm} \mathrm{dia}{ }^{-1}$ e $6,0 \mathrm{~mm} \mathrm{dia}{ }^{-1}$, respectivamente.

\section{CONCLUSÃO}

O algoritmo SEBAL aplicado a imagens do sensor TM Landsat 5 estimou de maneira coerente a partiçáo do balanço de energia em superficie. $O$ calor latente foi o componente de maior magnitude, especialmente para as regiôes de cultivo de soja, e que foi melhor representado pelo modelo quando comparado com medidas observacionais.

Os resultados mostram a potencialidade do SEBAL na geração de mapas da variabilidade espacial e temporal da evapotranspiração, dada a sensibilidade aos diferentes tipos de ocupação do solo, aos diferentes estádios de desenvolvimento da cultura da soja e às diferenças de demanda evaporativa da atmosfera. Assim, se aplicado a dados de alta resolução temporal e espacial, o algoritmo pode constituir importante ferramenta no monitoramento das necessidades hídricas das culturas.

\section{REFERÊNCIAS}

ALLEN, R.G.; PEREIRA, L.S.; RAES, D.; SMITH, M. Crop Evapotranspiration: Guidelines for computing crop water requirements. FAO, 1998. 300p. (FAO Irrigation and Drainage Paper, n.56).

ALLEN, R.G.; CLEMMENS, A.J.; BURT, C.M.; SOLOMON, K.; O'HALLORAN, T. Prediction accuracy for projectwide evapotranspiration using crop coefficients and reference evapotranspiration. Journal of Irrigation Drainage Engineering, v.131, p.24-36, 2005. http://dx.doi.org/10.1061/(ASCE)07339437(2005)131:1(24)

BALDOCCHI, D.D.; HICKS, B.B.; MEYERS, T.P. Measuring biosphere-atmosphere exchanges of biologically related gases with micrometeorological methods. Ecology, v.69, 1331-1340, 1988. http://dx.doi.org/10.2307/1941631

BASTIAANSSEN, W.G.M. SEBAL - Based sensible and latent heat fluxes in the irrigated Gediz Basin, Turkey. Journal of Hydrology, v.229, p.87-100, 2000. http://dx.doi.org/10.1016/S0022-1694(99)00202-4

BASTIAANSSEN, W.G.M.; MENENTI, M.; FEDDES, R.A.; HOLTSLAG, A.A.M. A remote sensing surface energy balance algorithm for land (SEBAL) I. Formulation. Journal of Hydrology, v.212, p.198212, 1998. http://dx.doi.org/10.1016/S0022-1694(98)00253-4

BASTIAANSSEN, W.G.M.; NOORDMAN, E.J.M.; PELGRUM, H.; DAVIDS, G. SEBAL model with remotely sensed data to improve water-resources management under actual field conditions. Journal of Irrigation and Drainage Engineering, v.131, p.85-93, 2005. http:// dx.doi.org/10.1061/(ASCE)0733-9437(2005)131:1(85)

BERLATO, M.A.; FONTANA, D.C. Variabilidade interanual da precipitaçáo e variabilidade dos rendimentos da soja no Estado do Rio Grande do Sul. Revista Brasileira de Agrometeorologia, v.7, p.119-125, 1999.

BERLATO, M.A.; MATZENAUER, R.; BERGAMASCHI, H. Evapotranspiração máxima da soja relaçôes com a evapotranspiração calculada pela equação de Penman, evaporação de tanque "classe A" e radiação solar global. Agronomia Sulriograndense, v.22, p.243259, 1986.

BEZERRA, B.G.; SILVA, B.B.; FERREIRA, N.J. Estimativa da evapotranspiração diária utilizando-se imagens digitais TM - Landsat 5. Revista Brasileira de Meteorologia, v.23, p.305-317, 2008. http:// dx.doi.org/10.1590/S0102-77862008000300005

CENTRO ESTADUAL DE METEOROLOGIA - CEMETRS. Atlas Climático do Rio Grande do Sul. Porto Alegre: Fundação Estadual de Pesquisa Agropecuária (FEPAGRO), 2011. v.1, 198p. Disponível em: <http://www.r3pb.com.br/atlas/Atlas_Climatico_do_Rio_Grande_ do_Sul/Atlas.html>. Acesso em: 27 jun. 2012.

CHANDER, G.; MARKHAM, B.L.; BARSI, J.A. Revised Landsat-5 thematic mapper radiometric calibration. IEEE Geoscience and Remote Sensing Letters, v.4, p.490-494, 2007. http://dx.doi.org/10.1109/ LGRS.2007.898285

COMPANHIA NACIONAL DE ABASTECIMENTO - CONAB. Comparativo de área, produção e produtividade safra 2011/2012. Disponível em: <http://www.conab.gov.br>. Acesso em: 10 maio 2012. 
FRENCH, A.N.; JACOB, F.; ANDERSON, M.C.; KUSTAS, W.P.; TIMMERMANS, W.; GIESKE, A.; SU, Z.; SU, H.; MCCABE, M.F.; LI, F.; PRUEGER, J.; BRUNSELL, N. Surface energy fluxes with the Advanced Spaceborne Thermal Emission and Reflection radiometer (ASTER) at the Iowa 2002 SMACEX site (USA). Remote Sensing of Environment, v.99, p.55-65, 2005. http://dx.doi.org/10.1016/j. rse.2005.05.015

GALVANI, E.; ESCOBEDO, J.F.; PEREIRA, A.B. Balanço de radiação e fluxo de calor no solo em ambiente natural e protegido cultivado com pepineiro. Bragantia, v.60, p.139-147, 2001. http:// dx.doi.org/10.1590/S0006-87052001000200010

JENSEN, J.R. Remote Sensing of the Environment: An Earth Resource Perspective. 2. ed. Upper Saddle River: Prentice Hall, 2007. 598p.

NEVES, B.V.B.; VERSIANI, B.R.; RODRIGUES, P.C.H. Geoprocessamento como ferramenta no estudo de correlação entre a dinâmica da cobertura vegetal e a evapotranspiração. Revista Brasileira de Recursos Hídricos, v.12, p.87-102, 2007.

RODRIGUES, J.O.; ANDRADE, E.M.; TEIXEIRA, A.S.; SILVA, B.B. Sazonalidade de variáveis biofísicas em regiôes semiáridas pelo emprego do sensoriamento remoto. Engenharia Agrícola, v.29, p.452465. 2009. http://dx.doi.org/10.1590/S0100-69162009000300012

SANTOS, T.V.; FONTANA, D.C.; ALVES, R.C.M. Avaliação de fluxos de calor e evapotranspiraçáo pelo modelo SEBAL com uso de dados do sensor ASTER. Pesquisa Agropecuária Brasileira, v.45, p.488496, 2010. http://dx.doi.org/10.1590/S0100-204X2010000500008
SILVA, B.B.; LOPES, G.M.; AZEVEDO, P.V. Balanço de radiação em áreas irrigadas utilizando imagens Landsat 5 - TM. Revista Brasileira de Meteorologia, v.20, p.243-252, 2005.

SILVA, B.B.; BEZERRA, M.V.C. Determinação dos fluxos de calor sensível e latente na superfície utilizando imagens TM - Landsat 5. Revista Brasileira de Agrometeorologia, v.14, p.174-186, 2006.

TANRÉ, D.; DEROO, C.; DUHAUT, P.; HERMAN, M.; MORCRETTE, J.J.; PERBOS, J.; DESCHAMPS, P.Y. Description of a computer code to simulate the satellite signal in the solar spectrum: the $5 \mathrm{~S}$ code. International Journal of Remote Sensing, v.11, p.659-668, 1990. http://dx.doi.org/10.1080/01431169008955048

VERSIANI, B.V.B.N.; RODRIGUES, P.C.H. Geoprocessamento como ferramenta no estudo de correlação entre a dinâmica da cobertura vegetal e a evapotranspiração. Revista Brasileira de Meteorologia, v.13, p.201-211, 2007.

WENG, Q.H.; LU, D.S.; SCHUBRING, J. Estimation of land surface temperature vegetation abundance relationship for urban heat island studies. Remote Sensing of Environment, v.89, p.467-483, 2004. http://dx.doi.org/10.1016/j.rse.2003.11.005

WEBLER, G.; ROBERTI, D.R.; CUANDRA, S.V.; COSTA, M.H. Evaluation of a Dynamic Agroecosystem Model (Agro-IBIS) for Soybean in Southern Brazil. Earth Interactions, v.16, p.1-15, 2012. http://dx.doi.org/10.1175/2012EI000452.1 